Mon. Not. R. Astron. Soc. 000,113(2010) Printed 27 October $2018 \quad$ (MN LATEX style file v2.2)

\title{
On Luminous Blue Variables as the Progenitors of Core-Collapse Supernovae, especially Type IIn Supernovae
}

\author{
V. V. Dwarkadas, ${ }^{1 \star}$ \\ ${ }^{1}$ Department of Astronomy and Astrophysics, U Chicago, 5640 S Ellis Ave, Chicago, IL 60637
}

\begin{abstract}
Luminous blue variable (LBV) stars are very massive, luminous, unstable stars that suffer frequent eruptions. In the last few years, these stars have been proposed as the direct progenitors of some core-collapse supernovae (SNe), particularly Type IIn $\mathrm{SNe}$, in conflict with stellar evolution theory. In this paper we investigate various scenarios wherein LBV stars have been suggested as the immediate progenitors of SNe. Many of these suggestions stem from the fact that the SNe appear to be expanding in a high density medium, which has been interpreted as resulting from a wind with a high mass-loss rate. Others arise due to perceived similarities between the SN characteristics and those of LBVs. Only in the case of SN $2005 \mathrm{gl}$ do we find a valid possibility for an LBV-like progenitor. Other scenarios encounter various levels of difficulty. The evidence that points to LBVs as direct core-collapse SNe progenitors is far from convincing. High mass-loss rates are often deduced by making assumptions regarding the wind parameters, which are contradicted by the results themselves. A high density need not necessarily imply a high wind mass-loss rate: wind shocks sweeping up the surrounding medium may give a high density shell with a low associated wind massloss rate. High densities may also arise due to wind clumps, or due to a previous LBV phase before the SN explodes as a Wolf-Rayet star. Some Type IIn SNe appear to signify more a phase in the life of a SN than a class of SNe, and may arise from more than one type of progenitor. A Wolf-Rayet phase that lasts for a few thousand years or less could be one of the more probable progenitors of Type IIns, and channels for creating short-lived W-R phases are briefly discussed.
\end{abstract}

Key words: circumstellar matter; stars: massive; stars: mass-loss; supernovae: individual: 2005gl; stars: winds, outflows; stars: Wolf-Rayet

\section{INTRODUCTION}

Core-collapse supernovae ( $\mathrm{SNe}$ ) arise from stars which have a zero-age main-sequence mass $\gtrsim 8 M_{\odot}$, and most likely $\gtrsim 11 M_{\odot}$. Surprisingly, the evolution of these stars towards the SN explosion, and their fate, is not well known after years of intensive research. Observational programs that set out to unearth the progenitors of $\mathrm{SNe}$ have so far conclusively detected around a dozen, almost all of which appear to be Type IIP SNe (Smartt 2009, and references therein), with red-supergiant (RSG) progenitors. The relationship of the remaining types of core-collapse $\mathrm{SNe}$ to their progenitors is inferred but not determined. It had generally been surmised that stars below about $30 M_{\odot}$ end their lives as RSGs, whereas those above $30 M_{\odot}$ become Wolf-Rayet (W-R) stars before they explode as SNe (Falk \& Arnett 1977; Podsiadlowski 1992).

\footnotetext{
* E-mail: vikram@oddjob.uchicago.edu
}

SN 1987A showed that blue supergiants (BSGs) could also be SN progenitors (Sonneborn et al. 1987), although it may take a binary companion to cause them to explode (Morris \& Podsiadlowski 2007; Podsiadlowski et al. 2007).

Type IIn SNe are relatively recent entrants to the SN classification scheme (Schlegel 1990). They are characterized by narrow emission lines (hence the ' $n$ ' designation) on a broad base, and often (but not always) show very strong $\mathrm{X}$-ray and radio emission. These characteristics suggest an interaction with a high-density medium, resulting in the high X-ray and radio flux. The high density has furthermore been attributed to a high mass-loss rate (Chugai et al. 2004; Gal-Yam \& Leonard 2009). That, combined with other considerations (Kotak \& Vink 2006; Gal-Yam et al. 2007; Vink 2008; Smith 2008b; Trundle et al. 2008, 2009) has led to suggestions that the progenitors of Type IIn SNe may be Luminous Blue Variables (LBVs). An LBV, according to Humphrevs \& Davidson (1994), is an "evolved, very luminous, unstable hot supergiant which suffers irregular erup- 
tions" or, in rare cases "giant eruptions like $\eta$ Car". The high mass-loss rate $\left(>10^{-4} M_{\odot} \mathrm{yr}^{-1}\right)$ during the eruptions is mainly what has prompted the suggestion that LBVs are Type IIn progenitors.

Stellar evolution theorists, on the other hand, have placed LBVs as a post-main sequence phase, but not as a final pre-SN phase (Schaller et al. 1992; Langer 1993; Langer et al. 1994; Stothers \& Chin 1996; Maeder et al. 2005; Maeder \& Meynet 2008). In conventional models of stellar evolution theory, the LBV phase may follow a mainsequence O-star phase or a H-rich WNL phase (Crowther 2008; Gräfener \& Hamann 2008), but is always succeeded by a H-poor WN phase or a WC phase. Thus stellar models require that the LBV star loses its $\mathrm{H}$ envelope, becomes a Wolf-Rayet (W-R) star and then explodes as a SN. Therefore they were not traditionally considered as progenitors of SNe. The high mass-loss rates deduced for some SNe such as 1994W (Chugai et al. 2004) and 2005gl (Gal-Yam \& Leonard 2009), as well as an explosive event that happened two years before the actual SN explosion in 2006jc (Foley et al. 2007), have prompted several authors to question whether LBV's could indeed be the immediate progenitors of SNe. This has led stellar evolution experts to explore whether $\mathrm{SNe}$ can explode while in the LBV stage. Several attempts are underway (Hirschi et al. 2010) but are limited by the paucity of knowledge regarding LBV and RSG mass-loss rates, and their metallicity dependence. Although possible scenarios have been put forward (Hirschi et al. 2010), it is fair to say that as of the writing of this paper, stellar models have so far not succeeded in having a star in the LBV stage end its life in a SN explosion.

This paper takes a closer look at some of the cases where LBVs have been proposed as the immediate progenitor of SNe. We explore the evidence that has been offered for LBV progenitors, and various reasons why other progenitors have been excluded. Our goal is to scrutinize various scenarios in detail to determine whether the evidence requires an LBV as the progenitor, or whether there is room for alternatives. Irrespective of whether stellar evolution theory currently allows for SNe to explode in the LBV stage, we wish to investigate whether the observations require that $\mathrm{SNe}$ explode in the LBV stage.

The plan of this paper is as follows: In $\$ 2$ we review proposed scenarios for an LBV star as the progenitor of a corecollapse SNe. We investigate aspects of the proposed scenario not considered by the authors, and examine whether the data are compatible with an LBV progenitor. 43 summarizes our results and outlines our suggestions, especially where Type IIn SNe are concerned.

\section{PROPOSED CASES FOR LBV PROGENITORS OF SNE}

\section{$2.1 \quad$ SN 2005gl}

This luminous type IIn SN is located in the nearby galaxy NGC 266. Pre-explosion Hubble Space Telescope (HST) images of the SN location led Gal-Yam et al. (2007) to suggest that a very bright point source with luminosity $L>10^{6} L \odot$ was the possible progenitor star of this SN. This identification was confirmed by Gal-Yam \& Leonard 2009, hereafter
GL09) when they observed that the putative progenitor had faded away and was no longer visible in $H S T$ images.

Spectra taken 8 days after discovery (between 8 and 33 days after explosion) show a narrow component to the $\mathrm{H} \alpha$ emission with a velocity of $420 \mathrm{~km} \mathrm{~s}^{-1}$, and an intermediate component with a velocity of about $1500 \mathrm{~km} \mathrm{~s}^{-1}$ (Gal-Yam \& Leonard 2009). No broad components representing typical SN ejecta velocities in the range of $10^{4} \mathrm{~km}$ $\mathrm{s}^{-1}$ is seen. Spectra on day 58 after discovery however do show a broad component with a $10^{4} \mathrm{~km} \mathrm{~s}^{-1}$ velocity. The intermediate component is missing. The narrow component on day 8 is interpreted by GL09 as the velocity of the unshocked progenitor wind. The velocity is deemed too high for red supergiants, and too low for Wolf-Rayet stars, but about right for an LBV star. The dense wind slows down the SN shock to about $1500 \mathrm{~km} \mathrm{~s}^{-1}$, whose velocity is reflected in the intermediate component. By day 58, according to GL09, the shock has crossed the dense wind and entered a region of less dense wind, and the emission is dominated by fast, unshocked ejecta. Assuming a steady LBV wind, GL09 determined a mass-loss rate of about $0.03 M_{\odot} \mathrm{yr}^{-1}$, which GL09 contend can only arise from an LBV star.

The large velocities observed in the day 58 spectrum attest to the fact that the ejecta have sufficient kinetic energy to expand with initial velocities $\gtrsim 10^{4} \mathrm{~km} \mathrm{~s}^{-1}$. Difficulties with this scenario could be associated with slowing down the fast-moving ejecta interacting with the ambient medium to about $1500 \mathrm{~km} \mathrm{~s}^{-1}$ in as little as 8 days, or as much as 33 days depending on the actual explosion date. We investigate various aspects of this scenario below.

Since the ejecta were expanding freely again on day 58 in this scenario, we estimate that they would have crossed the dense LBV wind in $50-75$ days or less (depending on explosion date). By day 8 they were already at $1500 \mathrm{~km} \mathrm{~s}^{-1}$, and would have slowed down even more, we may assume that the ejecta had an average velocity about $1250 \mathrm{~km} \mathrm{~s}^{-1}$ for the 50-75 days (depending on explosion date) that it took to cross the LBV wind medium. Since the velocity of the LBV wind was about $1 / 3$ that of the $\mathrm{SN}$, it means the wind in this phase would have lasted for about 3 times more, or about 150-225 days. Given the mass-loss rate of $0.03 M_{\odot}$ $\mathrm{yr}^{-1}$ calculated by GL09, this means that the total mass ejected in this wind was about $0.0125-0.0185 M_{\odot}$. This is an upper limit, as the wind phase could have conceivably lasted less than 50-75 days, and the average shock velocity could have been even lower (note the argument in the previous paragraph), given that it had slowed down from $>10,000$ to $1500 \mathrm{~km} \mathrm{~s}^{-1}$ in just 8-33 days.

If we make the extreme assumption that all the CS mass is contained in a thin, dense shell, the properties of the interaction have been shown to depend on the ratio of the mass of the shell to the mass of the ejecta (Tenorio-Tagle et al. 1990; Dwarkadas 2005). A ratio of 1 or higher is required for the shell to have a significant impact, while a ratio $<<1$ would have no impact as the ejecta would just plow through the shell. Dwarkadas (1997) also showed that the distribution of the mass, whether it is in a thin shell or spread out, is not as important as the amount of material. Therefore, in order to decelerate the ejecta completely, as seems likely, the ejecta mass involved in the interaction must be smaller than or comparable to $0.02 M_{\odot}$.

The important question therefore is how much ejecta is 
interacting with this amount of CSM material. This is difficult to say unless we can specify the ejecta mass and its distribution, and how the reverse shock expands into this ejecta, all of which are unknown. However, we can still make some estimates as to whether the scenario proposed is consistent with the observations. Following the arguments above, almost all the kinetic energy of the fraction of the ejecta interacting with the CSM is lost (the shock velocity decreases to $1 / 7$ the original, therefore the total energy decreases to $1 / 49$ the original). The total kinetic energy of the amount of ejecta interacting with the CSM was, at most, $0.5 \mathrm{M}_{e j} \mathrm{v}_{s i}^{2}$, where $\mathrm{v}_{s i}$ is the initial velocity of this material, which is at least $10,000 \mathrm{~km} \mathrm{~s}^{-1}$. The maximum luminosity that can be extracted from the radiative shock is $0.5 \dot{M} \mathrm{v}_{s}^{3} / v_{w}$. Given the mass-loss rate $\dot{M}=0.03 M_{\odot} \mathrm{yr}^{-1}$ and the wind velocity $v_{w}=420 \mathrm{~km}^{-1}$ deduced by GL09, the maximum luminosity that can be extracted immediately (when the shock velocity is $>10^{4} \mathrm{~km} \mathrm{~s}^{-1}$ ), is $4.5 \times 10^{43} \mathrm{ergs} \mathrm{s}^{-1}$. However, it is unlikely that the luminosity will be so high when the shock is moving so fast. On the other hand, the maximum luminosity on day $8(33)$ is $1.5 \times 10^{41} \mathrm{ergs} \mathrm{s}^{-1}$. (Note that Smith et al. (2010) find the wind velocity 34 days after discovery to be closer to $600 \mathrm{~km} \mathrm{~s}^{-1}$, which would reduce these numbers by about $2 / 3$ ). Since as the shock decelerates the amount of energy released would be expected to increase, we may assume that the average luminosity for the 8 (33) days was about 1. $\times 10^{42}$ ergs s$^{-1}$. Over 8 days, the total energy released is $6.9 \times 10^{47}$ ergs, while over 33 days it would be at most $2.85 \times 10^{48}$ ergs. The latter is probably an overestimate, as the shock deceleration would be slower over a larger period. Equating that to the total energy available for release gives the ejecta mass interacting with the CSM to be about 1-3 $\times 10^{-3} M_{\odot}$, and possibly up to a factor of 4 smaller. If the initial velocity is higher this mass increases somewhat, whereas if the wind velocity is closer to $600 \mathrm{~km} \mathrm{~s}^{-1}$ this decreases some.

The ejecta mass is an order of magnitude smaller than the mass in the LBV wind it is interacting with. Therefore deceleration of the ejecta is not an issue. We next check whether this amount of mass in the unshocked CSM is sufficient to provide the requisite $\mathrm{H} \alpha$ luminosity in the narrow line seen by GL09. We assume that the average velocity of the shock was about $2500 \mathrm{~km} \mathrm{~s}^{-1}$ over the first 8 days after discovery, or slightly lower if 33 days after explosion. Therefore, the maximum radius of the shock would be between about $1.7-7.1 \times 10^{14} \mathrm{~cm}$. This shock would therefore sweep up a mass of the CSM equal to at most $1.6 \times 10^{-2} M_{\odot}$. The mass of unshocked CSM remaining is about $2.5 \times 10^{-3} M_{\odot}$ or larger if the smaller time period is considered. Since the wind expanded for at most 225 days at $420 \mathrm{~km} \mathrm{~s}^{-1}$, it could have reached about $8.1 \mathrm{e} 14 \mathrm{~cm}$ (somewhat larger if the velocity was closer to $600 \mathrm{~km} \mathrm{~s}^{-1}$ ). The volume of unshocked wind is about $7.3 \times 10^{44} \mathrm{~cm}^{3}$. The luminosity of the $\mathrm{H} \alpha$ emission per unit volume can be approximately written as $\mathrm{E}_{H_{\alpha}}=\alpha n_{e}^{2} h \nu_{\alpha}$, where $\alpha=1-3 \times 10^{-13}$ is the recombination coefficient. The observed luminosity of $2.8 \times 10^{39}$ ergs $\mathrm{s}^{-1}$ in the narrow line gives the required $\mathrm{H}$ number density of the unshocked wind at that radius to be about $2 \times 10^{9}$ $\mathrm{cm}^{-3}$. We note that this is consistent with the actual number density of the unshocked wind just outside the shock. Furthermore the total mass of $\mathrm{H}$ required is smaller than the mass of unshocked wind. Given the many assumptions that have gone into this calculation, it appears reasonable that the unshocked wind has sufficient density to provide the required $\mathrm{H} \alpha$ luminosity. Note that if we take the expansion time as only 8 days then the density constraint is even easier to satisfy.

One final check to be made is whether the fast moving shock would become radiative immediately. We consider a shock of $11,000 \mathrm{~km} \mathrm{~s}^{-1}$ on day 1 . The cooling time for such a shock would be around 1 day at a density of $10^{10} \mathrm{~cm}^{-3}$, which is expected for the assumed numbers very close in to the star. Thus it seems likely that the shock would be a radiative shock, would be able to radiate the observed amount of energy in the appropriate time period, and that the ejecta mass would be small enough to be decelerated by the small amount of LBV material.

We note that the above calculations for the maximum luminosity were carried out assuming the existence of a steady wind, to be consistent with the assumptions of GL09, but without any supporting evidence for this assumption. As we show later in this paper, in many cases a steady wind is not supported by the observations. However, the above results could be derived in a different way. A lower limit to the available luminosity is set by the observed spectrum on day 8 , especially the $\mathrm{H} \alpha$ line luminosity. If we assume that the $\mathrm{H} \alpha$ luminosity is about a tenth of the total available luminosity, the other numbers follow accordingly. Therefore, even if one removes the steady wind constraint, the computed ejecta mass would not change much, and would still be lower than the calculated LBV mass. Removing the steady wind constraint would mean that we would not be able to easily calibrate the mass-loss rate. However, given the high density, which is required for the shock to be radiative (otherwise a broad $\mathrm{H} \alpha$ line would be seen on day 8), the remaining numbers would still be consistent. Also, in order for the shock wave to become radiative, a high density must exist from day 1 immediately outside the stellar envelope.

Although the calculations are necessarily approximate, and could be off by factors of 2-3, it appears that the interpretation of an extremely dense medium immediately surrounding the star may be consistent with most of the observed facts. The fact that the shock was no longer radiative in the spectrum taken on day 58 , given that the velocity must have been quite close to the initial velocity, suggests that the density in the wind into which the ejecta are now expanding must be lower by more than 2 orders of magnitude, to make the cooling time at that epoch larger than the flow time. If this is a wind from an LBV in quiescence, this seems reasonable, as the mass-loss rate will be at least two orders of magnitude lower. If this is from a prior phase, say a RSG wind, then since its velocity must be a factor of 20 or so smaller, this means that the mass-loss rate must be more than 3 orders of magnitude smaller, or of order $10^{-5} M_{\odot} \mathrm{yr}^{-1}$ or lower.

These calculations corroborate the scenario of the SN expanding in a very high density medium surrounding the star, with a mass-loss rate of about $0.03 M_{\odot} \mathrm{yr}^{-1}$ (assuming a steady wind) and a wind velocity between 420 and 600 $\mathrm{km} \mathrm{s}^{-1}$. GL09 suggest that these parameters are typical of LBVs and not found in other stars. While this is true, it must be noted that even in LBVs, such high mass-loss rates are only postulated for LBVs when they are undergoing an eruption, not when they are in a steady state. The detection 
of a very bright source $>10^{6} \mathrm{~L} \odot$ in roughly the $\mathrm{V}$-band is used by GL09 to support their assertion that this was no ordinary star. Does this definitely make it an LBV? Our knowledge of stellar evolution and the placement of stars on the $\mathrm{H}-\mathrm{R}$ diagram is incomplete, and it is unclear if every star with luminosity $>10^{6} \mathrm{~L}_{\odot}$ need necessarily be classified as an LBV star. LBVs are marked by their variability, which has not, and probably cannot, be demonstrated in this candidate star. Of concern also is the described evolutionary scenario that one is forced to accept. In this scenario, a star transitions into an LBV-like state, undergoes mass-loss with a mass-loss rate seen only in $\eta$ Car type eruptions for about 6 months or so, and then undergoes core-collapse. Did the star transition from a quiescent LBV into an eruptive state, and explode during, or immediately after, that state? Did the star transition from a different phase (say a red supergiant) to the LBV-like state, making it an LBV for only 6 months, during which time it underwent an eruption and then exploded as a SN? If the explosion of a star as an LBV is by itself a problem, the explosion of a star immediately after erupting as an LBV, with an extremely high mass-loss rate, is an even greater problem for stellar theorists to reconcile.

Therefore, many questions still remain unanswered. Given the available information, and with the above caveats, we currently rank SN2005gl as making an acceptable case for an LBV-like progenitor. Any alternative must have a high density wind (for the given velocity of around $500 \mathrm{~km} \mathrm{~s}^{-1}$ ) and a luminosity $>10^{6} \mathrm{~L}_{\odot}$ in the $\mathrm{V}$ band, and satisfy all the constraints computed above.

\subsection{Quasi-Periodic Modulations of Radio Supernovae}

Ryder et al. (2004) found modulations in the radio light curve of the Type IIb SN 2001ig, with a periodicity of 150 days (although this is based on only two peaks, with a probable third). Soderberg et al. (2006) report similar modulations in the radio lightcurve of SN 2003bg, at 120 and 300 days, with a probably third at 600 days. In this case it is not clear that they are periodic. Soderberg et al. (2006) attribute these to variations in the density of the CS medium. Kotak \& Vink (2006) suggest that these density variations may be due to LBV stars undergoing S Doradus type variations, with the density enhancements specifically due to the behavior at the bistability limit, which would lead to density jumps of a factor of 4 .

The reason for Kotak \& Vink (2006) suggesting LBV progenitors was to suggest a single star scenario rather than more contrived binary star scenarios. According to Kotak \& Vink (2006), the LBV scenario was in line with both the timescales as well as the amplitudes. However modelling of the light curves by Soderberg et al. (2006) has shown that density enhancements of factors of 1.8, 1.4 and 1.2 are required to fit the "bumps" in the light-curve. These are relatively small enhancements, and it is not clear why an LBV progenitor would need to be invoked to explain them, as was done by Kotak \& Vink (2006). In fact it is somewhat of a concern as to whether LBV outbursts would give rise to such low-level density enhancements; on the contrary, they will presumably lead to much larger changes in density than the $20-40 \%$ required to explain some of the lightcurve modulations. According to Kotak \& Vink (2006) themselves, the bistability jumps lead to a density enhancement of a factor of about 4. Mass-loss rates and velocities derived around the bistability jump in Vink et al. (1999) suggest perhaps even larger, not smaller, density enhancements, and no models suggest $10-20 \%$ enhancements. It is therefore unlikely that the density jumps due to S Doradus variations would match the modelled density enhancements. An exception would be if both the mass-loss rate and velocity enhancements were in the same direction, thus leading to a small enhancement in the density, which depends on their ratio. It should also be noted that the models by Soderberg et al. (2006) are approximate, and not based on hydrodynamical calculations. As they note, the self-similar solutions they have used are not truly applicable in a case where the density is increasing, thus leading to some uncertainty in the calculated results.

Even if bistability jumps were adequate, it is not clear that they should point only to an LBV. Although LBVs are the only objects in which bistability jumps have actually been observed, other massive stars, besides LBVs, could also experience these bistability jumps. Vink et al. (1999) have shown that the increase in mass-loss rate at the bistability jump is mainly due to radiative acceleration by Fe III, and therefore should occur in hot star atmospheres where this ion is present. And Vink et al. (2000) have derived massloss rates for all massive $\mathrm{O}$ and $\mathrm{B}$ stars assuming that they are affected by the bistability jumps.

An added consideration is that if the enhancements are due to changes in the wind parameters, then the wind-wind interaction will likely result in the sweeping up of the outer wind into a dense shell. This will increase the magnitude of the density enhancement, suggesting that the actual change in wind parameters may be even smaller, and making LBV progenitors even less likely.

The timescale calculation is uncertain as it relies on uncertain velocity determinations, as pointed out by Chevalier \& Soderberg (2010). Furthermore, 2003bg was initially classified as a Ic due to lack of hydrogen in the spectra. It is not known why a $\mathrm{H}$-rich $\mathrm{LBV}$ progenitor would give rise to a Ic spectrum. Perhaps the star transitioned into a W-R star just before explosion (\$3 explores such short W-R phases). According to the calculations of Chevalier \& Soderberg (2010) it would have had to happen on order of a quarter century before explosion. If this were true, the fast $\mathrm{W}-\mathrm{R}$ wind would be expected to sweep up the preceding LBV material into a thin dense shell, which is not seen. Also, if this were true, an LBV would not be the direct progenitor of the SN.

Chevalier \& Soderberg (2010) suggest that these SNe lie in a category called cIIb, or compact Type IIb, with radii of a few times $10^{11} \mathrm{~cm}$. They find that the progenitors of these $\mathrm{SNe}$ are less massive and more compact than LBVs, further discounting LBVs, and perhaps more suggestive of Wolf-Rayet (W-R) stars. Soderberg et al. (2006) suggested a single $\mathrm{W}-\mathrm{R}$ star progenitor with an average massloss rate of $3 \times 10^{-4} M_{\odot} \mathrm{yr}^{-1}$. However, this mass-loss rate is much higher than the known mass-loss rate of any single galactic W-R star (Crowther 2008). The combination of a high mass-loss rate and a short $\mathrm{W}-\mathrm{R}$ progenitor phase may suggest (see also 3.2 below) that the SN explosion occurred in a binary system (Eldridge et al. 2008). In fact Ryder et al. (2006) claim to have found a companion star, although Chevalier \& Soderberg (2010) argue that the star 
would have a weak wind and would not create the pinwheel like nebulae that Rvder et al. (2006) suggest gives the density variations. While certainly true, this does not preclude the dynamical effects of the binary companion. It is interesting to speculate whether the stellar orbit of the companion (rather than its wind), especially if it was quite eccentric, could affect the mass-loss from the star, leading to both an enhanced mass-loss rate and a variation in the mass-loss as the orbital radius changes.

In summary, although we can speculate on the progenitor, we are unable to pinpoint it. While strong arguments can be made against $\mathrm{S}$ Doradus type variations based on the modeling by Chevalier \& Soderberg (2010), there is also some uncertainty in the models themselves. These issues require further study and testing via both observations and modelling. Although we lean towards this scenario not providing sufficient evidence to suggest LBV supernova progenitors, a final assessment requires more observations than are currently available, accompanied by more detailed modelling.

\subsection{Type IIn SNe}

Many Type IIn SNe show signs of strong CS interaction, indicating that the SN shock may be encountering a medium of high density (Salamanca et al. 1998; Pastorello et al. 2002; Salamanca 2003; Chugai \& Danziger 2003; Chugai et al. 2004; Bauer et al. 2008), which has been interpreted as a sign of a high wind mass-loss rate. In order to investigate this assumption, it is useful to understand how the mass-loss rates have been calculated in several cases. If the medium into which the SN is expanding is a wind with a constant mass-loss rate $\dot{M}$ and wind velocity $v_{w}$, then the wind density decreases as $\mathrm{r}^{-2}$. The mass swept up by the expanding $\mathrm{SN}$ shock up to a radius $\mathrm{R}$ is the mass of the wind upto that radius

$M_{s w}=\dot{M} R / v_{w}$

The total energy in the swept-up material is

$E=0.5 M_{s w} v_{s}^{2}$

where $v_{s}$ is the shock velocity. Therefore, the kinetic energy dissipated per unit time, which is the maximum energy that can be extracted from the material over time, or the maximum attainable luminosity, is:

$L=d E / d t=0.5 \frac{\dot{M}}{v_{w}} v_{s}^{3}$

If the luminosity, shock velocity and the wind velocity are known, then this equation can be used to compute the massloss rate, with perhaps an unknown conversion efficiency factor added. Some form of this equation has been used to compute the mass-loss rate for several Type IIn SNe, including SN 1997ab (Salamanca et al. 1998), SN 2005gl, SN 2006gy (Smith et al. 2010), SN 2008iy (Miller et al. 2010), and SN 2006tf (Smith et al. 2008). The important point to note from the above derivation is that this equation is valid if, and only if, the wind mass-loss rate and velocity are constant with time, such that its density goes as $r^{-2}$, and the shock velocity is constant with tim 1. If this is not the case, then equation 1 is not valid, and neither are the succeeding equations, which of course means that equation 3 cannot be used. If it is the answer cannot be relied upon. In general, if the density profile is not at least as steep as $\mathrm{r}^{-2}$, application of these equations at a given radius will yield a misleadingly high mass-loss rate. Unfortunately, in many of the cases that they have been used, as illustrated below, the values derived contradict the assumptions of constant mass-loss rate and velocity, and an $\mathrm{r}^{-2}$ density profile.

For SN 2006gy, Smith et al. (2010) find, by using equation 3 , that the value of both $\dot{M}$ and $V_{C S M}$ is varying in time (their Figure 25). This is contrary to the assumptions inherent in the equation. Furthermore the SN blast wave is assumed to travel within this medium with a constant velocity, which is incompatible with the derived result of a medium with a varying mass-loss rate and velocity with time. It is almost impossible to construct a model in which the density parameter $w=\dot{M} / v_{w}$ varies non-uniformly by over a factor of 100 (as the authors find) while still keeping the velocity of the blast wave roughly constant. Their argument hinges on the untrustworthy computation of the wind density parameter from equation [3. As they themselves point out, their assumption that the entire luminosity comes from energy radiated behind a radiative shock is contrary to their own earlier assumption (Smith et al. 2007) of a shell-shocked diffusion model. If a substantial part of the luminosity arises from diffusion of energy from a shocked, optically thick shell, then this would lower their estimates considerably. Because of the inconsistencies in deriving these numbers, the total mass of swept-up circumstellar matter $(\mathrm{CSM})$ is also in doubt, and the high mass-loss rate questionable.

Miller et al. (2010) use equation 3 to compute the massloss rate in SN 2008iy. They assume a fixed wind velocity (although it is measured only at one epoch) and a constant blast wave velocity. They find that the mass-loss rate increases with time, then decreases. Given the increase in luminosity over time, they assert that the parameter $w$ must have increased over 400 days. However, if the parameter $w$ increases with time, the density profile no longer goes as $\mathrm{r}^{-2}$ over the 400 day rise period, the shock velocity should certainly not be constant, equation 1 is not valid, and neither are the others. Furthermore, although they assume a model of clumps in a rarefied wind to explain the late-time emission, they do not take into account any luminosity arising from shocks within the clumps in their mass-loss rate calculations, which could lower their estimates significantly. Thus the mass-loss rate is inconsistent with the assumptions used to obtain it, and therefore unreliable.

1 We note here for the sake of completeness that, if the shock is radiative, the formula

$$
L=2 \pi r^{2} \rho v^{3}
$$

can be used to compute the pre-shock density, assuming that all the energy is radiated away. This does not require $\mathrm{a}^{-2}$ medium, but it does require a radiative shock. However substituting $\rho=$ $\frac{\dot{M}}{4 \pi r^{2} v_{w}}$ to get the same equation as above does require assuming a constant mass-loss rate and wind velocity. All of the papers discussed here have assumed the medium goes as $\mathrm{r}^{-2}$ and used equation 3 to derive the mass-loss rate 
Miller et al. (2010) also compute the mass-loss rate by two other methods: (1) from the X-ray luminosity (Immler \& Kuntz 2005) and (2) from the H $\alpha$ luminosity. Unfortunately, the equation used to compute the mass-loss rate from the X-ray luminosity makes the same assumption, of the density of the medium decreasing as $\mathrm{r}^{-2}$, which is contravened by their results. Furthermore the authors use a low X-ray temperature of $10^{7} \mathrm{~K}$ to compute the cooling function at an age of 1.5 years, which is highly unlikely for a SN at such a young age, and incompatible with the high blast wave velocity of $5000 \mathrm{~km} \mathrm{~s}^{-1}$ that they have assumed, which would give a post-shock temperature of few times $10^{8}$ K. It is highly unlikely that the spectrum of such a complex dynamical region could be adequately described by one temperature, in any case. They have not taken into account the possibility that the X-ray emission could be arising from both the forward and reverse shocks. No attempt is made to take the SN deceleration into account when calculating the radius. Finally, we note that the numerical coefficient used in this equation is incorrect due to a missing factor of 4 (Immler et al. 2006).

In their computation of the mass-loss rate from the $\mathrm{H} \alpha$ luminosity, it is not clear what velocity Miller et al. (2010) refer to when using $V_{S N}$. Early in section 4.1 they refer to it as the velocity of the blast wave overrunning the CSM. This would then make this the velocity of the forward shock. In a medium as dense as the one they compute, the shock wave would sweep up the material and decelerate. The Chevalier (1982) self-similar solution gives the shock velocity as going as $t^{\alpha-1}$ where the deceleration parameter $\alpha<1$. The radius would then be given by $R_{S N}=V_{S N} t_{S N} / \alpha$; the authors have neglected the parameter $\alpha$. For the method of computing the mass-loss rate from the Halpha luminosity, they claim that $r_{1}$ is the "inner radius" corresponding to the position of the blast wave, but use the same formula $r_{1}=V_{S N} t_{S N}$, which is incompatible with their earlier assumption that this is the velocity of the blast wave overrunning the CSM. It was also assumed that a radius $r_{2}$ was the outer radius related to the fast-moving forward shock, and that $r_{2}>>r_{1}$. This is puzzling, because the outer shock is the one overrunning the CSM, which they earlier referred to as the blast wave. The assumption that $r_{2}>>r_{1}$ at day 711 would be incorrect in any model. The Chevalier (1982) solution predicts that the ratio of the outer to inner radii would be more like $20 \%$ in the self-similar case. In all cases they have neglected the value of $\alpha$ in their computations, which would make the radius at each epoch slightly larger.

In their scenario to explain the origin of the $400 \mathrm{~d}$ rise time for this SN, Miller et al. (2010) favor the clumpy wind scenario. As mentioned, it is then surprising that they do not take into account the luminosity from the radiative shocks in the clumps, which would reduce their mass-loss rate estimates. Miller et al. (2010) also argue against a wind-bubble scenario on the grounds that after peak the thin shell is overtaken by the ejecta, and the luminosity is powered by interaction with the ambient wind. This is not necessarily true. The shell could be thick, and the peak reached while the shock is still interacting with the shell. Even when the forward shock exits the shell and is interacting with the ambient wind, the reverse shock is still interacting with the shell for some time and could power the luminosity, and an extremely dense wind may not be needed. Finally, Miller et al.
(2010) say that addition of a wind-bubble component results in an unnecessary transition from an LBV to a W-R star. However, this is exactly the kind of scenario we envisage for many IIns in 3.4. Without detailed calculations, a wind-bubble scenario cannot be excluded. Due to the various questionable assumptions, their mass-loss estimates are untrustworthy, and the LBV scenario remains unproven.

In the case of SN 2006tf, the mass-loss rate derived by Smith et al. (2008), again using equation 3, exceeds $1 M_{\odot}$ $\mathrm{yr}^{-1}$, an astonishingly high rate. The mass-loss rates and wind density parameter are found to decrease gradually with time over 10 years, and decrease by a factor of 10 after that. Note that a mass-loss rate decreasing outwards, combined with a constant wind velocity, gives a density profile that decreases faster than $\mathrm{r}^{-2}$. This again means that the results are inconsistent with the assumptions (constant mass-loss rate and velocity) used to derive them. Smith et al. (2008) have assumed a shell-shocked diffusion model to explain the high luminosity, so it seems strange that they would calculate the mass-loss rate assuming that the entire luminosity arises from a radiative shock with $100 \%$ energy conversion (which is inconsistent with the constant shock speed assumed).

The mass-loss rate about 20 years prior to explosion is found to decrease by a factor of 10 , by analysis of the narrow lines. Assuming that the narrow lines arise from the CSM leads to the high estimate for the mass-loss rate and total mass. A plausible alternative may be that the narrow line emission arises from a very small section of the CSM, such as a denser clumpy component. The ratio of the narrow to broad line velocity of a factor of 10.5 suggests that the density contrast between the clumps and the surrounding medium would be about a factor of 110 . The narrow line velocity would not be the velocity of the CSM, but that of the shock within the clumps. Then the density of the medium is a factor of 100 lower and does not contribute to the emission, and the large mass-loss rate, and consequently large mass of the progenitor, are not necessary. The narrow and broad line components arise from different entities, and their behavior should not correlate, as is found for SN 2006tf. This model considerably diminishes the viability of the LBV hypothesis.

In the above cases, and others in the literature, the high mass-loss rate was the primary factor in suggesting an LBV progenitor. We have shown that the high mass-loss rates may be incorrectly interpreted. This does not mean that the density is not high - a high density can be achieved independently of a high mass-loss rate. In fact our main point is that a high density should not be used as a proxy for a high mass-loss rate, and should not therefore be seen as indicative of an LBV progenitor. This is elaborated on further in 3

\subsection{Circumstellar Nebulae}

The similarity in the ring structures of the nebula around the LBV star HD 168625, the B supergiant Sher 25, the star SBW1, and the nebula around SN 1987A led Smith (2007) to argue that the progenitor of SN 1987A may also have been an LBV. While the first object is known to be an LBV, the next two have abundances inconsistent with a RSG phase Smith 2008b)

An argument based purely on the shape of the CS 
structures is quite misleading. It is true that the shapes of nebulae around LBV stars and around SN 1987A are quite similar. However, it should be noted that the these shapes are also similar to that of several bipolar planetary nebulae, which form around low mass stars $2^{2}(<$ $\left.8 M_{\odot}\right)$. Just as $\mathrm{SNe}$ cannot be attributed to be arising from low mass progenitors on the basis of their circumstellar nebulae, they cannot be attributed to LBV progenitors purely on the basis of the shaping of their CS structures. The similarity in the shapes is a result of the similarity in the shaping mechanism, which is generally attributed to the interacting winds model and its variations (Kwok et al. 1978; Frank \& Mellema 1994; Dwarkadas et al. 1996; Dwarkadas \& Balick 1998; Balick \& Frank 2002), or due to an inherent asymmetry in the winds themselves (Frank et al. 1998; Dwarkadas \& Owocki 2002). The nebula around SN 1987A has been modelled in a manner similar to the models for PNe (Blondin \& Lundavist 1993). The details of the shaping mechanism may depend on the parameters, but the basic ideas are similar. This does not imply that the stars themselves are similar. Thus the similarity in shapes of the surrounding medium cannot be argued as a similarity in the progenitor star.

It could perhaps be argued that while the shaping mechanism may be effective, in the interacting winds model it does take a change in wind parameters to bring about this effect. In the case of SN 1987A, this requirement was fulfilled by the star evolving from the blue to the red side of the HR diagram and then back to the blue before exploding. The change in wind parameters, indeed the entire ring system, has been attributed to the transition from the RSG to the BSG stage (McCray 2007, and references within). If such red-blue loops were always required for the formation of the ring-like structures, this would set a timescale requirement which may be difficult to fulfill. However, we point out that such loops are but one way of achieving a change in the wind parameters. It has been reported that RSG and hypergiant stars may also show variability, related to changes in the wind parameters Humphrevs 2008; Levesque 2009), and may even undergo pulsations (Yoon \& Cantiello 2010), as described below. IRC+10420 and VY CMa both show complex and extensive circumstellar nebulae (Humphreys 2008). As discussed below, some stars may experience a brief Wolf-Rayet phase before explosion. Stars in binary systems can experience mass-loss episodes due to the pull of the secondary. Massive stars are known to have weak magnetic fields (Puls 2008), which can influence the wind, leading to disk formation and strong shocks, or at the very least density enhancements towards the magnetic equator (ud-Doula \& Owocki 2002). A spherical wind blowing into such a medium may lead to SN 1987Alike ring structures, as modelled by Blondin \& Lundavist (1993). And finally, a rotating star may create such shapes due to the latitudinal asymmetry of the wind from the star itself (Dwarkadas \& Owocki 2002). Given our still incomplete knowledge of stellar mass loss and evolution, it would be therefore misleading to attribute such shapes purely to LBVs without concrete evidence.

2 see catalog and images at http://www.astro.washington.edu/users/balick/PNIC/

\subsection{SN 2005gj}

Trundle et al. (2008) find multiple absorption component PCygni profiles of $\mathrm{H}$ and $\mathrm{He}$ in the spectrum of 2005gj. These profiles have earlier been noted by Prieto et al. (2007). Trundle et al. (2008) interpreted these as indicative of the progenitor's mass-loss history. Since such profiles have previously been seen only in LBVs, the natural assumption is to connect SN 2005gj to an LBV progenitor. The similarities in the wind velocity deduced from the profiles and those of LBVs was used to further support their argument.

The main problem with an LBV progenitor for SN $2005 \mathrm{gj}$ is, that in the papers describing its discovery and evolution (Aldering et al. 2006; Prieto et al. 2007), the type attributed to the SN was that of Type Ia. Prieto et al. (2007) did a cross-comparison with the SNID database, and the best comparison was overwhelmingly with a Type Ia spectrum. It is clear that SN 2005gj is not a typical Type Ia, since it shows Balmer line emission, whose width seems to be increasing with time. Trundle et al. (2008) appropriately note that the typical S II and Si II, which solidified the Ia status of the very similar SN 2002ic (since classified as a $\mathrm{Ia} / \mathrm{IIn}$ ), are not as clearly delineated in SN 2005gj. However, the overall resemblance of the spectrum of SN 2005gj to that of SN 2002ic, between days 26 and 84, is clearly shown in Prieto et al. (2007). The Ia-like features of SN 2002ic are even accepted by Trundle et al. (2008). Given this close resemblance between the two $\mathrm{SNe}$, it seems uncharacteristic that one would be a Ia and the other have an LBV progenitor. If it is a core-collapse SN, it may offer an explanation for the absorption components, but the spectrum becomes more difficult to explain.

The argument therefore boils down to whether SN $2005 \mathrm{gj}$ is a Type Ia SN or not. If it is then it cannot have a massive star LBV progenitor under any circumstances, and that assumption is not tenable. It is possible that the multiple absorption components rightly signify mass-loss, but possibly from a companion star, not the progenitor. Type Ia's are presumed to occur in a binary system, so the fact that material from the companion star may surround the progenitor is not unexpected. This could, and has been used to, explain the Balmer line emission in a Type Ia (Hamuv et al. 2003).

If it is shown to not be of Type Ia, then the LBV theory becomes more plausible. But it still needs direct proof that the profiles are due to the progenitor star as opposed to a companion. Also, it has to be shown that an LBV was actually the progenitor, as opposed to the fact that the progenitor star perhaps went through an LBV phase before losing its $\mathrm{H}$ and $\mathrm{He}$ envelope and undergoing a brief Wolf-Rayet phase (see 83 ) before explosion. The spectra presented by Prieto et al. (2007) do show some resemblance to a Type Ic spectrum at late times. Furthermore, one then has to explain the resemblance of the spectrum to that of other type Ia SNe, or at least to SN 2002ic, which would be difficult for a core-collapse event. Therefore, in the absence of further information, we remain agnostic towards the LBV interpretation. 


\section{DISCUSSION}

We have discussed in this paper various arguments put forward to suggest that luminous blue variable stars are progenitors of some core-collapse SNe. In many cases the high mass-loss rate that led to the suggestion of an LBV progenitor was based on the assumption that the medium into which the SN shock was expanding had a density profile decreasing as $\mathrm{r}^{-2}$, but the results show that the wind mass-loss parameter is variable with time, implying that the density profile does not decrease as $\mathrm{r}^{-2}$ but is more complicated. Applying an equation that works for only constant mass-loss rate and velocity winds to every possible situation, without evaluating whether the inherent assumptions are satisfied, leads to incorrect results. Furthermore, unless the density profile is decreasing even more steeply than $\mathrm{r}^{-2}$, application of these equations will generally yield a mass-loss rate that is higher than the true mass-loss rate.

The case of SN 2005gl is an exception. The lack of a broad emission line in the spectrum 8 days after discovery distinguishes it from many of the other Type IIn. We have examined quantitatively several aspects of the arguments made by GL09, confirming the basic structure of a highdensity medium surrounding the star. The identification of the progenitor star as having an exceptionally high luminosity in the $\mathrm{V}$ band (or close to it) is another argument in favor of an LBV candidate. However many questions still remain, such as whether an LBV-like star is the sole candidate for such a high-luminosity object, and why an LBV phase could have a high but steady mass-loss rate, resemblant of an LBV during eruption, for about 6 months, and then immediately explode as a SN. Given the currently available information, this could be considered a viable case for an LBV progenitor, with some unanswered questions remaining

The case for quasi-periodic modulations in the radio data is based on attributing those variations to S Doradus type instabilities. The arguments presented in this paper, when combined with those expressed in Chevalier \& Soderberg (2010), seem to contradict the suggestion of an LBV progenitor here from several different points of view. However more detailed models and further observations are required for a conclusive assesment. The morphological similarity of nebulae around LBV stars to those around SNe such as SN 1987A in no way implies similarity of the stars themselves, merely that of the shaping mechanism.

The case of SN 2005gj presents a quandry. It could be considered a plausible case for an LBV, with reservations, if it can be conclusively shown that the SN was not of Type Ia, but is not tenable if the Type Ia interpretation is true. The jury is still out on this one.

In summary, we have studied several suggested cases for LBV SN progenitors, and found that in only one case is there significant evidence to satisfy the interpretation of an LBV-like progenitor. While the difficulty in doing so may be considerable, it should be noted that in no case has variability, a hallmark of an LBV, actually been demonstrated, although it has been alluded to. We emphasize here that this is not an argument for or against the fact that stars can explode in the LBV stage, and thereby act as progenitors of $\mathrm{SNe}$. That is a discussion beyond the scope of this paper, and best left to stellar evolution theorists who are better equipped to answer that particular question. What we find here is that in only one proposed scenario is a strong case made to invoke an LBV-like object as the immediate progenitor.

Given our incomplete knowledge of stellar evolution, it is important to determine in the case of SN $2005 \mathrm{gl}$ whether other astronomical objects can have the required high luminosity in the appropriate band, or whether an LBV is the only candidate. The other arguments, although consistent and supportive of an LBV interpretation, are not conclusive on their own. Even one LBV progenitor, if confirmed, does call for a modification of stellar evolution theory. The challenge for stellar evolution theorists will then become more difficult, to explain not only why stars explode in the LBV stage, but whether they can do so immediately after a highmass loss rate phase.

At the same time, it appears that an LBV progenitor is not as pervasive as recent literature tends to suggest. It is clear that there are several Type IIn for which LBV progenitors, although suggested, are not necessary. This diversity in IIn progenitors provides some clues to their understanding, as discussed below.

\subsection{High Density CSM}

In many individual cases, the assertion of an LBV as a SN progenitor is made by noting that the observations require a high density CSM, and then assuming that the high density equates to a high mass-loss rate for the progenitor, typically $>10^{-2} M_{\odot} \mathrm{yr}^{-1}$, immediately preceding the $S N$ explosion. The high mass-loss rate is attributed to an $\eta$ Carinae type LBV explosion. It must be noted however, that even amongst LBVs, which are not very common in the first place, $\eta$ Car type explosive ejections are quite rare, with only two known events in the Galaxy - $\eta$ car itself in the 1840s, and $\mathrm{P}$ Cygni. In these extreme events, the star actually increases in luminosity during the outburst. In other LBVs with $\mathrm{S}$ Doradus type variations, which comprise the more common variety, the bolometric luminosity of the star does not vary (Puls et al. 2008). It remains to be seen if the frequency of explosive-type LBV events is high enough to account for all the SNe that are attributed to it.

Although we have shown that in many cases the high mass-loss rate argument may not hold, the high X-ray, radio or optical flux suggests that in many scenarios the CSM density close to the star must be high. It is therefore prudent to mention here that high density does not necessarily imply a high mass-loss rate. Other mechanisms can result in a high density medium. A common mechanism is the interaction of the fast wind from the star with the slower wind from a previous epoch, forming a wind-blown bubble. The fast wind sweeps the ambient medium or slower wind into a dense shell, whose density can be extremely high if the wind shock is radiative (see Figure 1), and the swept-up mass collapses into a very thin dense shell bordered by a radiative shock (Weaver et al. 1977; Dwarkadas 2005). The shell density and thickness depend on the exact wind parameters and interaction time, but the density can exceed $10^{4} \mathrm{~cm}^{-3}$. The mass in the dense shell comes from the swept-up material, not the progenitor wind, and could have been emitted over tens of thousands of years. Depending on the ambient density, the wind parameters and the time taken for the interaction, 
the size of the bubbles and the density enhancements may vary. A wind expanding supersonically for a sufficiently long time can lead to such dense shells, more-or-less independent of the mass-loss rate. The mass-loss rates cannot then be obtained from the density using the formulae described in 2.3

It is well known that as stars evolve, their wind parameters change, leading to the formation of large wind bubbles around the star (Garcia-Segura et al. 1996; Chu 2003, 2008; Freyer et al. 2003, 2006; Arthur 2007; Dwarkadas 2007a.d). Moderate changes in the wind parameters lead to the formation of smaller versions of these bubbles during the last few thousand years of a star's life. SN 1987A for example is surrounded by a wind bubble formed about 20,000 years before the SN explosion (McCray 2007). The progenitor's mass-loss rate must have been quite low, not exceeding $10^{-8} M_{\odot} \mathrm{yr}^{-1}$ (Chevalier \& Dwarkadas 1995; Lundqvist 1999; Dwarkadas $2007 \mathrm{c}$ b). The calculations of Dwarkadas et al. (2010) infer the presence of a wind bubble around SN $1996 \mathrm{cr}$, surrounded by a dense shell at about $0.03 \mathrm{pc}$.

Figure 1 shows the structure of such a bubble around a massive star, taken from a simulation of a Wolf-Rayet wind with mass-loss rate of $2.5 \times 10^{-6} M_{\odot} \mathrm{yr}^{-1}$ interacting with a RSG wind from a previous epoch (see for example Dwarkadas 2007d). Note that the density contrast between the density inside of the bubble and the shell is more than five orders of magnitude. The wind termination shock $\left(R_{t}\right)$, the contact discontinuity between the two winds $\left(R_{C D}\right)$ and the outer shock $\left(R_{o}\right)$ are marked. Significantly, in this structure, only the region labelled as free wind (to the left of the wind termination shock $\mathrm{R}_{t}$ and the dotted line) has a density profile that decreases as $\mathrm{r}^{-2}$. Equations 1 to 3 above can only be applied to this region to compute the wind parameters. If these equations are used to compute the density at other radii, especially in the dense shell region, it will result in a derived wind parameter that is extremely high, in a case where there is no freely expanding wind. This can mislead one into believing that the progenitor is a high mass-loss rate LBV star. The situation in a star with a varying mass-loss rate and wind velocity will be even more complicated.

A high density can also be achieved in localized regions due to high density clumps, which may be formed by hydrodynamical instabilities in the pre-existing circumstellar medium or in the ejected material. The interaction of the SN blast wave with a clump leads to a slow-moving shock expanding into the clump, and a reflected shock back into the inter-clump medium (Klein et al. 1994). The slow-moving shock velocity is represented in the formation of narrow lines (see 3.3 . The clumps, although high density and sufficient to give rise to the narrow lines in the spectrum, make only a minimal contribution to the total mass of the medium, are not representative of the overall density of the medium, and cannot be used to derive the global properties of the medium.

Finally, as current stellar evolution theory suggests for stars that are more massive than about $40 M_{\odot}$ (Hirschi et al. 2010), the possibility exists that the massive star experienced an LBV phase before becoming a Wolf-Rayet star. If the Wolf-Rayet stage is short enough, the dense LBV wind may be close in to the star, and/or may be sweptup by the faster W-R wind. Either of these possibilities will give rise to denser material in the surrounding medium, due to an LBV phase, but does not require the LBV to be the progenitor. This kind of scenario was suggested for SN 2001em by Chevalier (2007), following the models of Chugai \& Chevalier (2006), which showed that a dense medium around the SN may have formed about 2000 years earlier, with a mass-loss rate on order $10^{-3} M_{\odot \mathrm{yr}^{-1}}$. The star may have transitioned from an LBV to a W-R star shortly before explosion.

\subsection{Short Wolf-Rayet Phase}

In the case of SN 2001em, SN 1996cr, and many others (Dwarkadas et al. 2010, and references therein; also see (3.4), models suggest that the star explodes after a short $\mathrm{W}$-R phase $\left(<10^{4}\right.$ years $)$. Stellar evolution models, on the other hand, generally show that the W-R phase lasts for $>$ $10^{5}$ years. Under what conditions could we reasonably expect the star to remain in the $\mathrm{W}$-R phase for only a few thousand years or less before explosion? It turns out that there are several possibilities. (1) One way this could happen is if the star has a binary companion. Eldridge et al. (2008) have shown that stars in a binary system may enter the $\mathrm{W}-\mathrm{R}$ phase at much lower initial mass as compared to single stars. The stars at the very low end of the mass range of stars able to become $\mathrm{W}$-R stars will have very short W-R lifetimes (see Figures 2 and 3 in Eldridge et al. 2008), on order tens of thousands of years or less. In principle, the same short lifetime would apply to single stars which are at the lower end of the mass range where they can become $\mathrm{W}-\mathrm{R}$ stars, although since the cutoff mass to become a W-R is higher, the number of stars is much smaller. (2) Binary interaction can result in Case $\mathrm{C}$ mass transfer which follows the He burning phase. Under certain conditions this can lead to explosive common-envelope ejection (Podsiadlowski 2007; Podsiadlowski et al. 2010), which leads to the ejection of both the hydrogen and helium layers late in the evolution of the star. This results in a W-R star with a natural $10^{4}$ year or less timescale. (3) Another promising channel for the formation of a short duration W-R stage was recently shown by the work of Yoon \& Cantiello (2010), which followed up on earlier work by Heger et al. (1997). They find that RSGs could have strong pulsation driven superwinds. These would cause the RSG to just remove its outer layers and become a W-R star in the last hundreds to thousands of years of evolution. Such pulsation-driven winds may also result in the formation of circumstellar shells around the star, and thus may be useful in understanding Type IIns. In fact, Fransson et al. (2002) suggested that Type IIn SNe, and especially SN 1995N, could arise from RSG progenitors which experience superwinds, a scenario that resembles the one outlined above (although they did not specifically advocate a $\mathrm{W}-\mathrm{R}$ progenitor).

Overall it seems that several channels exist which can result in short-duration Wolf-Rayet phases, and therefore it is reasonable to propose that some $\mathrm{SNe}$ can experience a short W-R phase before explosion. Many scenarios that require a short $\mathrm{W}-\mathrm{R}$ phase tend to indicate stars with initial mass that is lower than the minimum single-star mass required to become a W-R star. If some fraction of Type IIn arise from such lower mass W-R stars, then it may indicate that, contrary to the general assumption, IIns do not arise from very massive stars but instead from a lower av- 


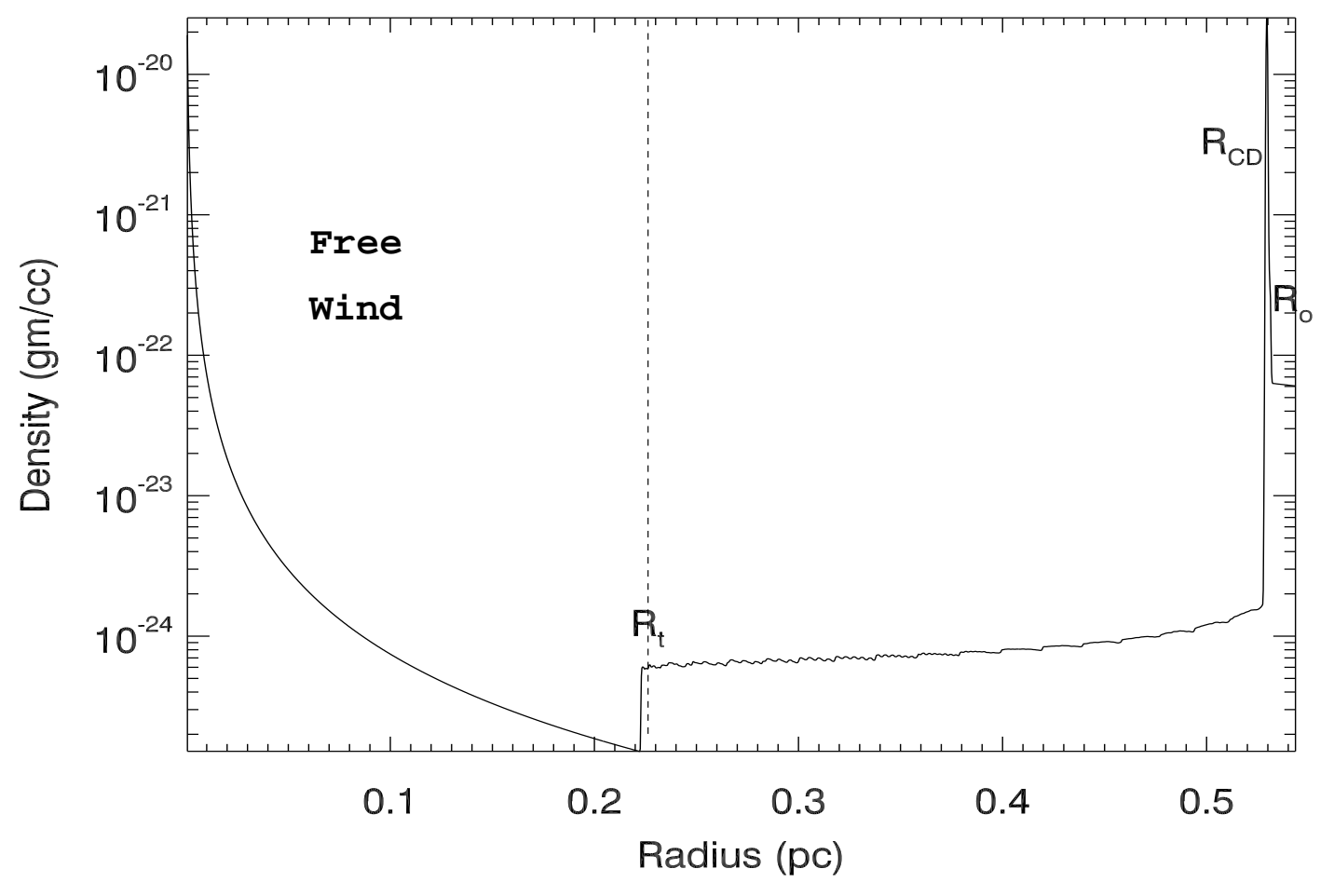

Figure 1. The density profile in a wind-blown bubble around a high-mass star. The bubble was formed by a Wolf-Rayet wind, with a mass-loss rate of $2.5 \times 10^{-6} M_{\odot} \mathrm{yr}^{-1}$ and a wind velocity of $2500 \mathrm{~km} \mathrm{~s}^{-1}$, interacting with a RSG wind from a previous epoch. The interaction gives rise to a low-density bubble surrounded by a high density shell. This is one way to get a high density in the surrounding medium without have an extremely high mass-loss rate wind. In this figure, the region marked as "Free Wind" denotes the freely expanding wind, to which equations 1 to 3 can be applied. Application of these equations at other radii could result in very large, but incorrect, mass-loss rates.

erage initial mass population. A similar result was derived by Anderson \& James (2008), who found, from an investigation of the association of the explosion sites of Type IIn SNe with recent star formation, that the majority of Type IIns do not arise from the most massive stars, which would be the case if they all had LBV progenitors.

\subsection{Velocities indicated by Narrow Emission lines}

In many of the SNe discussed, and several others, narrow lines seen in the spectrum are invoked as arising from the unshocked, ionized CSM. In some cases, the presence of P-Cygni absorption components may indicate the presence of an outflow. Their velocity is interpreted as the velocity of the ambient wind, and is considered intermediate between that of a RSG and a W-R but appropriate for LBVs (Trundle et al. 2008). However, it is not certain what appropriate velocities are for LBVs. Trundle et al. (2008) state that LBV velocities lie between $50-300 \mathrm{~km} \mathrm{~s}^{-1}$, Kotak \& Vink (2006) suggest velocities between 100 and 500 $\mathrm{km} \mathrm{s}^{-1}$, (Smith et al. 2010) find $600 \mathrm{~km} \mathrm{~s}^{-1}$ from their spectrum of SN 2005gl appropriate for an LBV, and $\eta$ Carinae has reported velocities exceeding $3000 \mathrm{~km} \mathrm{~s}^{-1}$ (Smith 2008a). Furthermore, such velocities are not unique to LBVs.
Blue supergiants (BSGs) are also known to have velocities of a few hundred $\mathrm{km} \mathrm{s}^{-1}$. The circumstellar medium close in to SN 1987A has been modelled with a BSG wind velocity between 300-600 $\mathrm{km} \mathrm{s}^{-1}$ (Blondin \& Lundqvist 1993; Chevalier \& Dwarkadas 1995; Dwarkadas 2007c)

As discussed above there are other equally plausible alternatives for the narrow emission lines. In the cases of SN 1986J (Chugai 1993), SN 1988z (Chugai \& Danziger 1994), SN 1978K (Chugai et al. 1995) and SN 1996cr (Dwarkadas et al. 2010) at least, lines of narrow or intermediate width are explained as arising from the interaction of the SN shock with a clumpy medium. The FWHM of these lines denotes the velocity of the shock driven into the clumps. They are unrelated to the velocity of the surrounding medium. There also exists the possibility that the narrow line spectrum is contaminated by emission from the host galaxy. We note that clumps could also provide the high density for much of the emission. Interestingly, in the case of SN 1996cr, both a dense shell (to provide the enhanced emission) and dense clumps in the ambient wind (to explain the narrow lines) have been postulated by Dwarkadas et al. (2010). 


\subsection{Type IIn SNe}

The Type IIn SN class is most likely to be associated with LBV progenitors. However Type IIn's are not a homogeneous group, and in fact not all of them show strong radio emission van Dvk et al. 1996) or X-ray emission. This may suggest that not all Type IIn are undergoing CS interaction with a high density medium. The diversity of IIns makes it inappropriate to classify IIns, or the similarly designated Type Ibn or Icn, as a separate class of SNe. Some are more indicative of a phase in the lifetime of a $\mathrm{SN}$, where it is interacting with high density material, and which it will outgrow in a matter of years or decades, no matter what class they fall in or the nature of their progenitor. A similar point had been made earlier by Kotak et al. (2004) with regards to the dual classification of SN 2002ic as a Ia/IIn. The fact that Type Ia's sometimes share a dual classification as Type IIns typifies the inhomogeneity among the group, and their lack of a common progenitor.

Many IIns earn the ' $n$ ' designation later on in their lifetime, when they are presumably interacting with high density material and are visible. But this merely emphasizes a feature in the late-time spectrum rather than a physical property of the progenitor. Mainly it is the initial designation of type which would be useful in pinpointing a progenitor. This is a small but subtle distinction, because unlike other SNe classes, it suggests that Type IIn's need not arise from a single progenitor (if in fact other subtypes do arise from a single progenitor, which has not been shown). The fact that IIns could have both high and low initial mass progenitors is consistent with this statement.

A scenario of a wind bubble blown by a W-R or blue supergiant wind was suggested for the Type IIn SN 1996cr by Dwarkadas et al. (2010), with the mass-loss rate of the progenitor star being $<10^{-4} M_{\odot} \mathrm{yr}^{-1}$, and in all probability substantially less. The Type Ibn SN 2006jc was suggested by Foley et al. (2007) to be a Wolf-Rayet star exploding in a dense He-rich medium formed about two years before the star exploded. Pastorello et al. (2008) propose that the progenitor of the hybrid SN 2005la was a very young Wolf-Rayet (WN-type) star which experienced mass ejection episodes shortly before core collapse. SN 2003bg was initially classified as a Ic before becoming a IIn, while SN 2001em transformed from a Ib/c to a IIn, possibly undergoing substantial mass-loss about 1000-2000 years before explosion (Chugai \& Chevalier 2006). Stockdale et al. (2010) indicate that SNe 1996aq and $2004 \mathrm{dk}$, both classified as Type Ic after explosion, are evolving in a similar manner to SN $1996 \mathrm{cr}$ and SN 2001em.

These considerations suggest that a subset of SNe with the ' $n$ ' designation may show a wind-bubble structure on parsec or even sub-parsec scales, due to a change in the wind parameters close to the end of the star's life. We propose that one channel for the formation of Type IIns that show enhanced late-time X-ray and radio luminosity may be W-R stars with fast winds, where the final W-R phase lasts for a few thousand years or less (see $\$ 3.2$ ). The $\mathrm{W}-\mathrm{R}$ wind sweeps up the surrounding medium, leading to the formation of dense shells close in to the star. The interaction of the SN shock wave with this medium gives rise to many of the notable properties of Type IIn SNe, including the large X-ray and radio luminosity at late times, while obviating the need for a high mass-loss rate progenitor (the mass-loss rates for Galactic W-R stars are $<10^{-4.4} M_{\odot} \mathrm{yr}^{-1}$ (Crowther 2008)).

There are some Type IIns that show IIn features almost from the time of explosion, including SN 2005ip (Smith et al. 2009) and SN 1995N (Fransson et al. 2002). In these $\mathrm{SNe}$ it appears that a high density region sits just outside the stellar envelope. SN 2005gl also shows an extremely high density region outside the stellar envelope, but as seen it does not extend far out radially. These are all indicative of a wide diversity in this class of objects, and would probably require a different channel for their formation. SN $2005 \mathrm{gl}$ has been associated with an LBV-like progenitor. Using Spitzer spectroscopy, Fox et al. (2010) have studied SN 2005ip, and conclude that a progenitor eruption formed a dust shell about 100 years prior to explosion. They find that a large mass-loss rate $>10^{-2} \mathrm{M}_{\odot} \mathrm{yr}^{-1}$ is required to explain this dust shell, and therefore suggest that it too is consistent with an LBV progenitor. On the other hand, Smith et al. (2009) found a mass-loss rate 2-3 orders of magnitude lower. Their optical observations do not require, or invoke, a dust shell, and they assumed that the mass loss arises in a steady wind with density decreasing as $\mathrm{r}^{-2}$. Smith et al. (2009) concluded that the progenitor was a RSG star, despite having a wind velocity of about $120 \mathrm{~km} \mathrm{~s}^{-1}$. It is interesting to speculate whether these two arguments can perhaps be reconciled by invoking pulsationdriven superwinds in RSGs (Yoon \& Cantiello 2010), which could potentially give rise to the high mass-loss rate dust shell just prior to explosion.

Follow-up multi-wavelength observations with increased frequency, specifically in the radio and X-ray regimes, will undoubtedly reveal more Type IIn SNe. Regular monitoring may detect more analogs of SN 1996cr, with X-ray and radio emission increasing over year-long periods of time. Such observations, accompanied by detailed theoretical investigations including hydrodynamic modelling and multiwavelength emission computations, are urgently needed, if we are to determine the nature and progenitors of this interesting category of objects.

\section{ACKNOWLEDGMENTS}

I gratefully acknowledge discussions with Alex Heger and Georges Meynet, who as usual patiently answered my queries on stellar evolution, and directed me to various literature regarding the formation of Wolf-Rayet stars with a short lifetime. I thank Roger Chevalier for reading an earlier version of this manuscript and providing suggestions that helped to improve it. I also wish to acknowledge a very comprehensive report by the anonymous referee, which considerably helped to improve and strengthen the arguments in this paper. VVD's research is supported by grants TM9-0001X, TM9-0004X, GO9-0086B, and GO0-11095B, provided by the National Aeronautics and Space Administration through Chandra Awards issued by the Chandra $\mathrm{X}$-ray Observatory Center, which is operated by the Smithsonian Astrophysical Observatory for and on behalf of the National Aeronautics Space Administration under contract NAS8-03060. I thank my collaborators in our research on SN 1996cr, Dan Dewey and Franz Bauer, which led me to look much more closely at LBV progenitors. 


\section{REFERENCES}

Aldering G., Antilogus P., Bailey S., Baltay C., Bauer A., Blanc N., Bongard S., Copin Y., Gangler E., Gilles S., Kessler R., Kocevski D., Lee B. C., Loken S., Nugent P., Pain R., 2006, ApJ, 650, 510

Anderson J. P., James P. A., 2008, MNRAS, 390, 1527

Arthur S. J., 2007, in Revista Mexicana de Astronomia y Astrofisica Conference Series Vol. 30 of Revista Mexicana de Astronomia y Astrofisica Conference Series, WindBlown Bubbles and HII Regions around Massive Stars. pp 64-71

Balick B., Frank A., 2002, ARAA, 40, 439

Bauer F. E., Dwarkadas V. V., Brandt W. N., Immler S., Smartt S., Bartel N., Bietenholz M. F., 2008, ApJ, 688, 1210

Blondin J. M., Lundqvist P., 1993, ApJ, 405, 337

Chevalier R. A., 1982, ApJ, 258, 790

Chevalier R. A., 2007, in Revista Mexicana de Astronomia y Astrofisica Conference Series Vol. 30 of Revista Mexicana de Astronomia y Astrofisica Conference Series, Circumstellar Interaction Around Type Ib/c Supernovae and the GRB Connection. pp 41-48

Chevalier R. A., Dwarkadas V. V., 1995, ApJL, 452, L45

Chevalier R. A., Soderberg A. M., 2010, ApJL, 711, L40

Chu Y., 2003, in K. van der Hucht, A. Herrero, \& C. Esteban ed., A Massive Star Odyssey: From Main Sequence to Supernova Vol. 212 of IAU Symposium, Ring nebulae around massive stars throughout the Hertzsprung-Russell diagram. pp 585-+

Chu Y., 2008, in F. Bresolin, P. A. Crowther, \& J. Puls ed., IAU Symposium Vol. 250 of IAU Symposium, Bubbles and Superbubbles: Observations and Theory. pp 341-354 Chugai N. N., 1993, ApJL, 414, L101

Chugai N. N., Blinnikov S. I., Cumming R. J., Lundqvist P., Bragaglia A., Filippenko A. V., Leonard D. C., Matheson T., Sollerman J., 2004, MNRAS, 352, 1213

Chugai N. N., Chevalier R. A., 2006, ApJ, 641, 1051

Chugai N. N., Danziger I. J., 1994, MNRAS, 268, 173

Chugai N. N., Danziger I. J., 2003, Astronomy Letters, 29, 649

Chugai N. N., Danziger I. J., della Valle M., 1995, MNRAS, 276, 530

Crowther P. A., 2008, in F. Bresolin, P. A. Crowther, \& J. Puls ed., IAU Symposium Vol. 250 of IAU Symposium, Properties of Wolf-Rayet Stars. pp 47-62

Dwarkadas V. V., 1997, PhD thesis, UNIVERSITY OF VIRGINIA

Dwarkadas V. V., 2005, ApJ, 630, 892

Dwarkadas V. V., 2007a, ApSS, 307, 153

Dwarkadas V. V., 2007b, in Immler S., McCray R., eds, American Institute of Physics Conference Series Vol. 937 of American Institute of Physics Conference Series, SN Shock Evolution in the Circumstellar Medium surrounding SN 1987A. pp 120-124

Dwarkadas V. V., 2007c, in Revista Mexicana de Astronomia y Astrofisica Conference Series Vol. 30 of Revista Mexicana de Astronomia y Astrofisica Conference Series, Supernova Explosions in Winds and Bubbles, with Applications to SN 1987A. pp 49-56

Dwarkadas V. V., 2007d, ApJ, 667, 226

Dwarkadas V. V., Balick B., 1998, ApJ, 497, 267
Dwarkadas V. V., Chevalier R. A., Blondin J. M., 1996, ApJ, 457, 773

Dwarkadas V. V., Dewey D., Bauer F., 2010, ArXiv e-prints

Dwarkadas V. V., Owocki S. P., 2002, ApJ, 581, 1337

Eldridge J. J., Izzard R. G., Tout C. A., 2008, MNRAS, 384, 1109

Falk S. W., Arnett W. D., 1977, ApJS, 33, 515

Foley R. J., Smith N., Ganeshalingam M., Li W., Chornock

R., Filippenko A. V., 2007, ApJL, 657, L105

Fox O. D., Chevalier R. A., Dwek E., Skrutskie M. F., Sugerman B. E. K., Leisenring J. M., 2010, ArXiv e-prints Frank A., Mellema G., 1994, ApJ, 430, 800

Frank A., Ryu D., Davidson K., 1998, ApJ, 500, 291

Fransson C., Chevalier R. A., Filippenko A. V., Leibundgut B., Barth A. J., Fesen R. A., Kirshner R. P., Leonard D. C., Li W., Lundqvist P., Sollerman J., Van Dyk S. D., 2002, ApJ, 572, 350

Freyer T., Hensler G., Yorke H. W., 2003, ApJ, 594, 888

Freyer T., Hensler G., Yorke H. W., 2006, ApJ, 638, 262

Gal-Yam A., Leonard D. C., 2009, Nature, 458, 865

Gal-Yam A., Leonard D. C., Fox D. B., Cenko S. B., Soderberg A. M., Moon D.-S., Sand D. J., Li W., Filippenko A. V., Aldering G., Copin Y., 2007, ApJ, 656, 372

Garcia-Segura G., Mac Low M.-M., Langer N., 1996, AA, 305, 229

Gräfener G., Hamann W., 2008, AA, 482, 945

Hamuy M., Phillips M. M., Suntzeff N. B., Maza J., González L. E., Roth M., Krisciunas K., Morrell N., Green E. M., Persson S. E., McCarthy P. J., 2003, Nature, 424, 651

Heger A., Jeannin L., Langer N., Baraffe I., 1997, AA, 327, 224

Hirschi R., Meynet G., Maeder A., Ekström S., Georgy C., 2010, in C. Leitherer, P. Bennett, P. Morris, \& J. van Loon ed., Astronomical Society of the Pacific Conference Series Vol. 425 of Astronomical Society of the Pacific Conference Series, Stellar Evolution in the Upper HR Diagram. pp $13-+$

Humphreys R. M., 2008, in F. Bresolin, P. A. Crowther, \& J. Puls ed., IAU Symposium Vol. 250 of IAU Symposium, The Evolutionary State of the Cool Hypergiants Episodic Mass Loss, Convective Activity and Magnetic Fields. pp 111-118

Humphreys R. M., Davidson K., 1994, PASP, 106, 1025 Immler S., Brown P. J., Milne P., The L., Petre R., Gehrels N., Burrows D. N., Nousek J. A., Williams C. L., Pian E., Mazzali P. A., Nomoto K., Chevalier R. A., Mangano V., Holland S. T., Roming P. W. A., Greiner J., Pooley D., 2006, ApJL, 648, L119

Immler S., Kuntz K. D., 2005, ApJL, 632, L99

Klein R. I., McKee C. F., Colella P., 1994, ApJ, 420, 213

Kotak R., Meikle W. P. S., Adamson A., Leggett S. K., 2004, MNRAS, 354, L13

Kotak R., Vink J. S., 2006, AA, 460, L5

Kwok S., Purton C. R., Fitzgerald P. M., 1978, ApJL, 219, L125

Langer N., 1993, Space Science Reviews, 66, 365

Langer N., Hamann W.-R., Lennon M., Najarro F., Pauldrach A. W. A., Puls J., 1994, AA, 290, 819

Levesque E., 2009, ArXiv e-prints

Lundqvist P., 1999, ApJ, 511, 389

Maeder A., Meynet G., 2008, in A. de Koter, L. J. Smith, 
\& L. B. F. M. Waters ed., Mass Loss from Stars and the Evolution of Stellar Clusters Vol. 388 of Astronomical Society of the Pacific Conference Series, Mass Loss and the Evolution of Massive Stars. pp 3-+

Maeder A., Meynet G., Hirschi R., 2005, in T. G. Barnes III \& F. N. Bash ed., Cosmic Abundances as Records of Stellar Evolution and Nucleosynthesis Vol. 336 of Astronomical Society of the Pacific Conference Series, Chemical Abundances and Yields from Massive Stars. pp 79-+ McCray R., 2007, in S. Immler, K. Weiler, \& R. McCray ed., Supernova 1987A: 20 Years After: Supernovae and Gamma-Ray Bursters Vol. 937 of American Institute of Physics Conference Series, Supernova 1987A at Age 20. pp 3-14

Miller A. A., Silverman J. M., Butler N. R., Bloom J. S., Chornock R., Filippenko A. V., Ganeshalingam M., Klein C. R., Li W., Nugent P. E., Smith N., Steele T. N., 2010, MNRAS, 404, 305

Morris T., Podsiadlowski P., 2007, Science, 315, 1103

Pastorello A., Quimby R. M., Smartt S. J., Mattila S., Navasardyan H., Crockett R. M., Elias-Rosa N., Mondol P., Wheeler J. C., Young D. R., 2008, MNRAS, 389, 131

Pastorello A., Turatto M., Benetti S., Cappellaro E., Danziger I. J., Mazzali P. A., Patat F., Filippenko A. V., Schlegel D. J., Matheson T., 2002, MNRAS, 333, 27

Podsiadlowski P., 1992, PASP, 104, 717

Podsiadlowski P., 2007, Royal Society of London Philosophical Transactions Series A, 365, 1163

Podsiadlowski P., Ivanova N., Justham S., Rappaport S., 2010, MNRAS, 406, 840

Podsiadlowski P., Morris T. S., Ivanova N., 2007, in S. Immler, K. Weiler, \& R. McCray ed., Supernova 1987A: 20 Years After: Supernovae and Gamma-Ray Bursters Vol. 937 of American Institute of Physics Conference Series, The progenitor of SN 1987A. pp 125-133

Prieto J. L., Garnavich P. M., Phillips M. M., DePoy D. L., Parrent J., Pooley D., Dwarkadas V. V., Baron E., Bassett B., Becker A., Cinabro D., DeJongh F., Dilday B., Doi M., Frieman J. A., Hogan C. J., Holtzman J., Jha S., Kessler R., 2007, ArXiv e-prints

Puls J., 2008, in F. Bresolin, P. A. Crowther, \& J. Puls ed., IAU Symposium Vol. 250 of IAU Symposium, Physical and Wind Properties of OB-Stars. pp 25-38

Puls J., Vink J. S., Najarro F., 2008, AApR, 16, 209

Ryder S. D., Murrowood C. E., Stathakis R. A., 2006, MNRAS, 369, L32

Ryder S. D., Sadler E. M., Subrahmanyan R., Weiler K. W., Panagia N., Stockdale C., 2004, MNRAS, 349, 1093

Salamanca I., 2003, in E. Perez, R. M. Gonzalez Delgado, \& G. Tenorio-Tagle ed., Star Formation Through Time Vol. 297 of Astronomical Society of the Pacific Conference Series, The Dense Circumstellar Material Around Type IIn Supernovae. pp 429-+

Salamanca I., Cid-Fernandes R., Tenorio-Tagle G., Telles E., Terlevich R. J., Munoz-Tunon C., 1998, MNRAS, 300, L17

Schaller G., Schaerer D., Meynet G., Maeder A., 1992, AApS, 96, 269

Schlegel E. M., 1990, MNRAS, 244, 269

Smartt S. J., 2009, ARAA, 47, 63

Smith N., 2007, AJ, 133, 1034

Smith N., 2008a, Nature, 455, 201
Smith N., 2008b, in F. Bresolin, P. A. Crowther, \& J. Puls ed., Massive Stars as Cosmic Engines Vol. 250 of IAU Symposium, Episodic Mass Loss and Pre-SN Circumstellar Envelopes. pp 193-200

Smith N., Chornock R., Li W., Ganeshalingam M., Silverman J. M., Foley R. J., Filippenko A. V., Barth A. J., 2008, ApJ, 686, 467

Smith N., Chornock R., Silverman J. M., Filippenko A. V., Foley R. J., 2010, ApJ, 709, 856

Smith N., Li W., Foley R. J., Wheeler J. C., Pooley D., Chornock R., Filippenko A. V., Silverman J. M., Quimby R., Bloom J. S., Hansen C., 2007, ApJ, 666, 1116

Smith N., Silverman J. M., Chornock R., Filippenko A. V., Wang X., Li W., Ganeshalingam M., Foley R. J., Rex J., Steele T. N., 2009, ApJ, 695, 1334

Soderberg A. M., Chevalier R. A., Kulkarni S. R., Frail D. A., 2006, ApJ, 651, 1005

Sonneborn G., Altner B., Kirshner R. P., 1987, ApJL, 323, L35

Stockdale C., Heim M., Vandrevala C. M., Rentz B. T., Weiler K. W., Panagia N., Van Dyk S. D., Bauer F. E., Immler S., Sramek R. A., Pooley D., Ryder S. D., Marcaide J., 2010, in Bulletin of the American Astronomical Society Vol. 41 of Bulletin of the American Astronomical Society, Using Radio and X-ray Observations to Explore Supernovae with Evolving Optical Classifications. pp 360$+$

Stothers R. B., Chin C.-W., 1996, ApJ, 468, 842

Tenorio-Tagle G., Bodenheimer P., Franco J., Rozyczka M., 1990, MNRAS, 244, 563

Trundle C., Kotak R., Vink J. S., Meikle W. P. S., 2008, AA, 483, L47

Trundle C., Kotak R., Vink J. S., Meikle W. P. S., 2009, in G. Giobbi, A. Tornambe, G. Raimondo, M. Limongi, L. A. Antonelli, N. Menci, \& E. Brocato ed., American Institute of Physics Conference Series Vol. 1111 of American Institute of Physics Conference Series, Can LBV's Be The Direct Progenitors of Core Collapse Supernovae?. pp 311-314

ud-Doula A., Owocki S. P., 2002, ApJ, 576, 413

van Dyk S. D., Weiler K. W., Sramek R. A., Schlegel E. M., Filippenko A. V., Panagia N., Leibundgut B., 1996, AJ, 111,1271

Vink J. S., 2008, New Astronomy Review, 52, 419

Vink J. S., de Koter A., Lamers H. J. G. L. M., 1999, AA, 350,181

Vink J. S., de Koter A., Lamers H. J. G. L. M., 2000, AA, 362,295

Weaver R., McCray R., Castor J., Shapiro P., Moore R., 1977, ApJ, 218, 377

Yoon S., Cantiello M., 2010, ApJL, 717, L62

This paper has been typeset from a $\mathrm{TE}_{\mathrm{E}} \mathrm{X} / \mathrm{L} \mathrm{L}_{\mathrm{E}} \mathrm{X}$ file prepared by the author. 